\title{
Visually Guided Movements Suppress Subthalamic Oscillations in Parkinson's Disease Patients
}

\author{
Ramin Amirnovin, Ziv M. Williams, G. Rees Cosgrove, and Emad N. Eskandar \\ Department of Neurosurgery, Massachusetts General Hospital, Harvard Medical School, Boston, Massachusetts 02114
}

There is considerable evidence that abnormal oscillatory activity in the basal ganglia contributes to the pathogenesis of Parkinson's disease. However, little is known regarding the relationship of oscillations to volitional movements. Our goal was to evaluate the dynamics of oscillatory activity at rest and during movement. We performed microelectrode recordings from the subthalamic nucleus (STN) of patients undergoing deep brain stimulation surgery. During recordings, the patients used a joystick to guide a cursor to one of four targets on a monitor. We recorded 184 cells and 47 pairs of cells in 11 patients. At rest, 26 cells $(14 \%)$ demonstrated significant oscillatory activity, with a mean frequency of $18 \mathrm{~Hz}$. During movement, this oscillatory activity was either reduced or completely abolished in all of the cells. At rest, 18 pairs (38\%) of cells in five patients exhibited synchronized oscillatory activity, with a mean frequency of $15 \mathrm{~Hz}$. In 17 of the 18 pairs, both of the cells exhibited oscillations, and, in one pair, only one of the cells was oscillatory. These synchronized oscillations were also significantly decreased with movement. There was a strong inverse correlation between firing rates and oscillatory activity. As the firing rates increased with movement, there was a decrease in oscillatory activity. These findings suggest that visually guided movements are associated with a dampening and desynchronization of oscillatory activity in STN neurons. One possible explanation for these observations is that the increased cortical drive associated with movement preparation and execution leads to a transient dampening of STN oscillations, hence facilitating movement.

Key words: Parkinson's disease; subthalamic nucleus; oscillations; neurons; visual guidance; movement

\section{Introduction}

Parkinson's disease (PD) is characterized by the classic triad of rigidity, akinesia, and tremor (Lang and Lozano, 1998a). One of the more intriguing aspects of $\mathrm{PD}$ is the dynamic nature of the symptoms. For example, akinetic patients can frequently overcome their inability to move in response to compelling visual stimuli (Glickstein and Stein, 1991). A potentially related observation is that the tremor of PD occurs primarily at rest but is attenuated with movement (Lang and Lozano, 1998a). However, the mechanisms underlying these observations remain unclear.

In $\mathrm{PD}$, the loss of dopaminergic input into the basal ganglia results in dysregulation of the motor pathways, resulting in abnormal activity of the subthalamic nucleus (STN) and globus pallidus internus (GPi), among other structures (Albin et al., 1989; DeLong, 1990). Based on these findings and a growing body of clinical data, the GPi and STN are now the preferred targets for the surgical treatment of PD (Lang and Lozano, 1998b; DBS Study Group, 2001). Options for treatment include placing a deep brain stimulation (DBS) electrode in either nucleus or ablating a portion of the GPi (pallidotomy), with the goal of reduc-

\footnotetext{
Received Aug. 6, 2004; revised Nov. 3, 2004; accepted Nov. 4, 2004.

This work was supported by National Institutes of Health Grant NS41851, by a grant from the Rappaport Foundation, and by a grant from the Parkinson's Disease Foundation.

Correspondence should be addressed to Dr. Emad Eskandar, Department of Neurosurgery, Massachusetts General Hospital, 15 Parkman Street, Ambulatory Care Center-021, Boston, MA 02114-3117. E-mail: eeskandar@partners.org.

DOI:10.1523/JNEUROSCI.3242-04.2004

Copyright $\odot 2004$ Society for Neuroscience $\quad$ 0270-6474/04/2411302-05\$15.00/0
}

ing or modifying abnormal neuronal activity (Lang and Lozano, 1998b; Alkhani and Lozano, 2001; DBS Study Group, 2001). At most centers, microelectrode recordings are used to localize the target nuclei, and hence these surgeries provide a unique but under-used opportunity to evaluate neuronal activity in human subjects (Hutchison et al., 1998).

Studies using microelectrode recordings from the 1-methyl4-phenyl-1,2,3,6-tetrahydropyridine (MPTP) primate model of PD (Bergman et al., 1994) and from PD patients (Levy et al., 2000) have demonstrated an apparent increase in oscillatory and synchronized activity in STN neurons. These abnormalities have been posited to play a role in the pathogenesis of $\mathrm{PD}$ in a number of ways. For example, the abnormal oscillations may contribute to parkinsonian tremor (Bergman et al., 1994; Levy et al., 2000). Additionally, abnormal oscillation and increased synchronization may either block the normal flow of information through the basal ganglia or lead to a loss of neuronal selectivity and hence result in the observed impairments in movement (Boraud et al., 2000; Levy et al., 2001). However, the relationship of oscillatory activity to actual movements has not been extensively studied. Primates treated with MPTP are usually unable to perform behavioral tasks (Raz et al., 2000). Neuronal activity in humans undergoing surgery is typically evaluated using passive limb movements or relatively unconstrained voluntary movements, neither of which allow a careful assessment of neuronal activity relative to movement (Abosch et al., 2002). Therefore, to characterize the dynamics of the oscillatory activity, we recorded from the STN of PD patients undergoing subthalamic DBS surgery 
while they performed a structured visual guidance task (Eskandar and Assad, 2002).

\section{Materials and Methods}

Patient selection. All of the subjects included in this study had idiopathic Parkinson's disease for $>4$ years, a Hoehn and Yahr score of three or higher, and a documented response to L-3,4-dihydroxyphenylalanine replacement therapy. All of the patients had a preoperative neurological exam with detailed information on their tremor and PD symptoms. Subjects were excluded from surgery if they had cognitive impairment, active psychiatric disorders, or anatomic abnormalities on magnetic resonance imaging. None of the patients had undergone previous surgery for the treatment of PD. Informed consent for participation in the study was obtained in accordance with a protocol approved by the Massachusetts General Hospital Institutional Review Board. The decision to perform surgery was made based on clinical indications and was not related to participation in this study.

Electrophysiology. In all of the subjects, antiparkinsonian medications were withheld starting at midnight before the surgery. No sedatives were given during the surgery. The general techniques of stereotactic localization and intraoperative microelectrode recordings were described previously (Hutchison et al., 1998; Eskandar et al., 2000). We performed physiologic localization using an array of three tungsten microelectrodes separated by $2 \mathrm{~mm}$ and placed in a parasagittal orientation. The electrodes were advanced simultaneously in $50 \mu \mathrm{m}$ increments using a motorized drive. Amplification of the neuronal signal and control of the microdrive were handled by a dedicated intraoperative system (Alpha Omega Engineering, Nazareth, Israel). Neuronal activity was bandpass filtered $(300 \mathrm{~Hz}$ to $6 \mathrm{kHz})$ and sampled at $20 \mathrm{kHz}$. The behavioral paradigm was controlled by a Macintosh G4 computer (Apple Computers, Cupertino, CA). The neuronal, behavioral, and electromyographic (EMG) data were stored on a third computer equipped with a Cambridge Electronics Design (Cambridge, UK) system.

EMG activity was recorded at $1000 \mathrm{~Hz}$ using tin-disk surface electrodes to ensure broad and early detection of activity (Lee and Assad, 2003). We recorded EMG activity primarily from the biceps and triceps. Early on, we made recordings from the forearms, biceps, triceps, and deltoids. We found that the biceps and triceps had the earliest, largest, and most reliable activation. These results are similar to those obtained in primates performing a nearly identical task (Lee and Assad, 2003).

Anatomic localization. During surgery, microelectrode recordings were used to localize the STN. The subthalamic nucleus has characteristic high firing rates compared with the surrounding structures and has clear dorsal and ventral borders that are evident when reconstructing neuronal activity along the electrode trajectories. Cells used for analysis were recorded primarily from the dorsolateral motor subterritory of the STN based on stereotactic localization, reconstruction of the electrode trajectories, and the presence of audible responses to passive or spontaneous limb movements. When the electrodes were in the motor territory of the STN, we began recording at relatively regular $0.25 \mathrm{~mm}$ increments.

Behavioral paradigm. Once the microelectrodes were in the STN, the subjects viewed a computer monitor and performed the behavioral task by moving a joystick with the contralateral hand. The joystick was mounted such that movements were in a horizontal orientation with the elbow flexed at $\sim 45^{\circ}$. Each trial began with the presentation of a small central fixation point (see Fig. $1 \mathrm{~A}$ ). After a brief delay $(250 \mathrm{msec})$, four small gray targets appeared arrayed in a circular manner around the fixation point. After a $1500 \mathrm{msec}$ delay, a randomly selected target turned green. At this point, the subject used the joystick to guide a cursor from the center of the monitor toward the green target. Once the target was reached, a tone sounded, indicating that the subject had successfully completed the task, and the stimuli were erased. Patients were required to reach the target within $5 \mathrm{sec}$ of the green cue presentation, although they typically reached the target within 1 sec of the onset of the stimulus. There was an intertrial interval of $1500 \mathrm{msec}$. Subjects were required to return the joystick to the center position before a new trial started. If the subject prematurely moved the joystick, strayed beyond the confines of an invisible corridor, failed to reach the target, or failed to return the joystick to its central position, the trial was aborted and excluded from analysis. The directions were pseudorandomized to ensure an equal number of trials in each direction. Patients typically performed 12-24 correct trials in each direction at a given site. Each recording run lasted $\sim 3-5 \mathrm{~min}$.

Analysis. Spikes were sorted using a template-matching algorithm refined by principal component analysis and cluster cutting (Harris et al., 2000). Interspike interval histograms and autocorrelograms were computed for all of the spike trains (see Fig. 1C). Given the high cellular density of the STN, it was not possible to obtain perfect isolations in all cases. Seventy percent of our cells were relatively well isolated, with a firing rate of $<100 \mathrm{~Hz}$ (see Fig. 1C). This group included a spectrum of isolation, in which some cases had clear single units, and others represented multiunit recordings that were dominated by a single cell. The remaining $30 \%$ of the units had a firing rate of $>100 \mathrm{~Hz}$ and represented multiunit recordings that could not be refined further (see Fig. 1D). Because our goal was to study the relative changes of the firing rate and the oscillatory activity of the cells, we included both of the groups in our analysis. Rasters and peristimulus histograms were constructed for all of the recorded neurons and were aligned on the start of hand movement, as determined by an initial $1^{\circ}$ deflection of the joystick (from a possible range of $18^{\circ}$ ). The spike trains were divided further into epochs when the subject was at rest and epochs when the subject was actively moving the joystick.

Autocorrelograms (1 msec bins) were computed for each spike train, and cross-correlograms (CCs) were computed for pairs of simultaneously recorded spike trains at rest and during movement. Periodic oscillations were detected using the correlograms of all of the recorded spike trains, calculated for a $500 \mathrm{msec}$ offset with a bin size of $1 \mathrm{msec}$. The power spectra (PS) of the spike trains were calculated by the Fourier transform of the autocorrelograms (after removing the trough at time 0 ), allowing $1 \mathrm{~Hz}$ resolution of the frequencies (Raz et al., 2000). Additional analysis was performed between 5 and $40 \mathrm{~Hz}$. The signal-to-noise ratio (SNR) was calculated using the following formula: ((max power of the peak) - (mean power of the PS between 5 and $40 \mathrm{~Hz})$ )/(SD of the PS between 5 and $40 \mathrm{~Hz}$ ). Significant oscillatory activity was defined as a power spectra peak exhibiting an SNR greater than three times the SD of the PS (Raz et al., 2000).

The baseline firing rate and SD of the CCs were computed over the first and last $250 \mathrm{msec}$ of the CC. The CC was considered significant if more than three consecutive $\mathrm{CC}$ bins straddling the center differed from the baseline by 2.5 SD (Raz et al., 2000). If the CC was significant and a peak in its PS had an SNR greater than three times the SD, the pair of cells was considered to have synchronized oscillatory activity. The relationship between oscillatory activity and firing rate was analyzed by dividing the spike trains into $250 \mathrm{msec}$ bins and computing the average firing rate and the power of the dominant oscillatory frequency for each bin.

\section{Results}

\section{Oscillatory activity and movement}

Across all of the subjects, the average time to complete a single movement was $730 \pm 100 \mathrm{msec}$. During an experiment, subjects performed several hundred trials, and hence there were a large number of movement epochs. The resting epochs were selected from the intervening delay periods when the subject was not moving. We adjusted the length of the resting epoch used in the analysis so that both epochs had approximately an equal number of spikes. In this way, we could ensure that differences in the estimate of the oscillatory activity were not attributable to differences in the number of spikes analyzed. On average, the resting epochs were $900 \pm 100 \mathrm{msec}$. Across spike trains, the mean number of spikes analyzed in each of the two epochs was $4900 \pm 460$ spikes.

A total of 184 cells were isolated from the STN of 11 subjects. Twenty-six cells (14\%) demonstrated significant oscillatory activity at rest. The mean frequency of oscillation at rest was $18 \pm 3$ $\mathrm{Hz}(11.7-25.4 \mathrm{~Hz})$. A representative extracellular recording is displayed in Figure $1 B$. At rest, there is pronounced oscillatory 
A
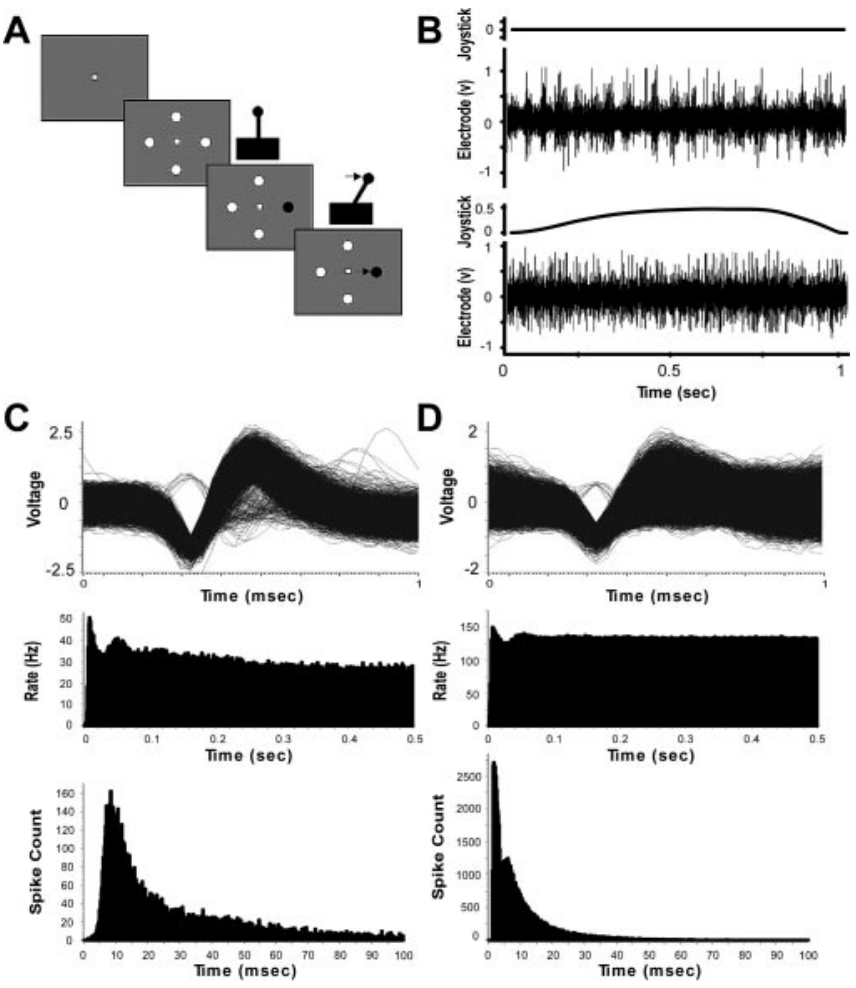

Figure 1. A, Behavioral task. $B$, Neuronal recordings from the STN with subjects' respective joystick movements at rest and during movement. The top traces in each panel represent deflection of the joystick along one axis. C, D, Representative action potentials (top), autocorrelogram (middle), and interspike interval histogram (bottom) for a relatively well isolated unit and a multiunit recording.

activity, which abates completely while the movement is underway.

Of these 26 units, 18 (69\%) were relatively well isolated (Fig. $1 C)$. The remaining eight cells (31\%) exhibited higher firing rates and did not exhibit a clear refractory period (Fig. 1D). However, their properties were not otherwise appreciably different from the other recordings.

The observation regarding the attenuation of oscillatory activity by movement was confirmed in the power spectral analysis. Representative autocorrelograms and power spectra at rest and during movement for a relatively well isolated unit are demonstrated in Figure $2 \mathrm{~A}$. This unit had significant power at $11.7 \mathrm{~Hz}$ during rest that was nearly abolished with movement. Figure $2 C$ demonstrates the autocorrelograms and power spectra of a higher firing frequency recording that exhibits the same phenomenon. There is significant power at $19.5 \mathrm{~Hz}$ at rest, which abates with movement. The sloping correlograms may be attributable to a slow drift in certain situations. However, the resting and movement epochs were alternating continuously, and hence any drift would have affected both equally.

The decrease in the power of oscillatory activity with movement was present across the population of cells (Fig. $3 A$ ). There were no units in which oscillatory activity significantly increased during movement. Overall, $38 \%$ of oscillatory cells ceased to exhibit significant oscillations during movement, and the remaining $62 \%$ exhibited a significant decrease in the power of oscillatory activity. Accordingly, the average power of oscillatory activity across the cells was diminished significantly during movement compared with that at rest (paired $t$ test; $p<0.05$ ) (Fig. 3B).

\section{Synchronized activity and movement}

Forty-seven pairs of cells were recorded from 11 patients. Analysis of the cross-correlograms revealed that 18 of these pairs (38\%) in five patients had significant synchronized oscillatory activity. The mean frequency of synchronized oscillatory activity was $15.4 \pm 3 \mathrm{~Hz}(11.7-21.5 \mathrm{~Hz})$ at rest. A representative crosscorrelogram of one such pair of cells is displayed in Figure $2 B$. This pair of cells exhibited significant power at $11.7 \mathrm{~Hz}$ during rest, which decreased significantly during movement. Another example from a higher-frequency recording demonstrates the same effect (Fig. 2D). The average power of synchronized activity among pairs of neurons exhibited a significant reduction during movement (paired $t$ test; $p<0.05$ ) (Fig. $3 B$ ). In 17 of the 18 pairs, both of the cells exhibited oscillations, and, in one pair, only one of the two cells had significant oscillatory activity. In comparison, 26 pairs of cells (55\%) demonstrated synchronized activity without overt oscillations. None of these cells exhibited a change in synchrony during movement.

\section{Oscillatory activity and firing rates}

Oscillatory cells had a mean baseline firing rate of $58 \pm 3 \mathrm{~Hz}$, which was significantly higher than that of nonoscillatory cells $(51 \pm 2 \mathrm{~Hz} ; t$ test; $p<0.001)$. This is consistent with previous primate studies demonstrating that the oscillatory cells found in the STN of MPTP-treated monkeys exhibited increased baseline firing rates compared with nonoscillatory cells (Bergman et al., 1994). Across the population of oscillatory cells, the average firing rate began changing significantly from baseline 750-500 msec before the onset of movement (Fig. $3 C$ ). This change in firing rate preceded the first change in EMG activity, which on average began $\sim 200 \mathrm{msec}$ before the overt movement. The firing rate reached its maximum in the $250 \mathrm{msec}$ period before the onset of movement, at which point the power of oscillation was at its minimum (Fig. 3C). There was a relatively tight significant inverse linear correlation between neuronal firing rate and oscillatory activity. Thus, as the firing rate of a given cell increased in conjunction with movement, the power of oscillations decreased $(r=-0.84 ; p<0.001)$ (Fig. 3D).

\section{Oscillations and tremor}

All of the patients had a preoperative neurological exam incorporating a rating of their tremor (Table 1, None, Mild, Moderate, Severe). The 26 oscillatory cells were found in 7 of the 11 patients. Of these seven patients, three had little or no tremor, three had mild resting tremor, and only one had a severe tremor. Of the four patients whose cells did not have oscillatory activity, one had little or no tremor, two had a mild tremor, and one had severe tremor. These tremor ratings were obtained during outpatient assessment. However, during the actual performance of the task, the patients clinically exhibited relatively little tremor, and there was no evidence of tremor on EMG. Thus, there did not seem to be a clear relationship between the severity of the observed resting tremor and the presence of oscillatory activity.

\section{Discussion}

Performing physiologic experiments in human subjects undergoing surgery presents unique constraints and difficulties not encountered in the laboratory setting. Nevertheless, it is perhaps the only way to evaluate and hopefully understand the dynamics of neuronal activity in true idiopathic PD. Animal models of PD using neurotoxins such as MPTP are not perfectly analogous to the human disease, and treated animals are usually unable to perform complex behavioral tasks (Raz et al., 2000). Hence, it is 
A

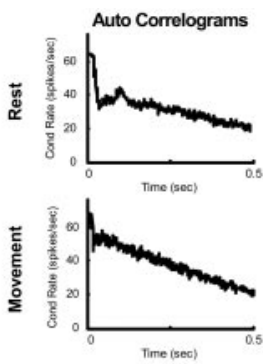

B
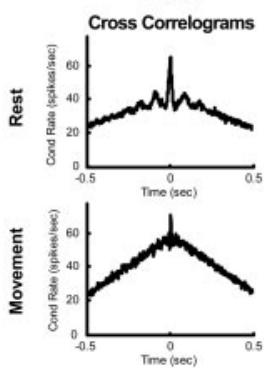
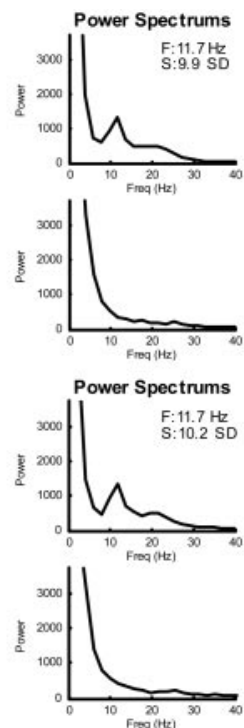

C

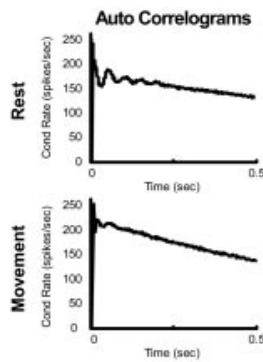

D

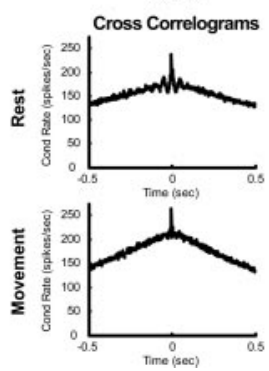

Figure 2. $A-D$, Representative autocorrelograms $(A)$ and cross-correlograms $(B)$ during movement and at rest with their respective power spectra from a relatively well isolated unit and from a multiunit recording $(C, D)$. Note the presence of a slope at the edges of the correlograms, caused by a slow drift in the recorded firing rates. The frequency ( $F$, Freq) of the statistically significant peaks and their SNRs (S) are shown in the top right corners of the power spectra. Cond Rate, Conditional rate, the rate at which the firing from one cell relates to itself (autocorrelogram) or another cell (CC).

A

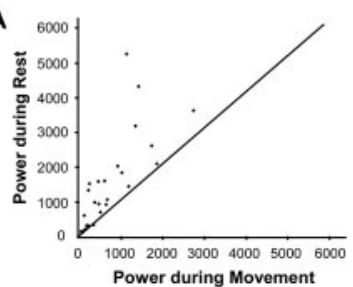

B

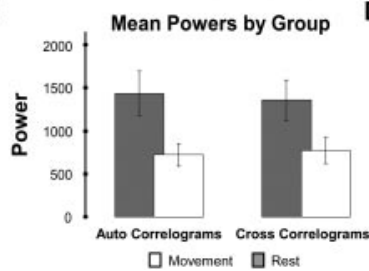

D
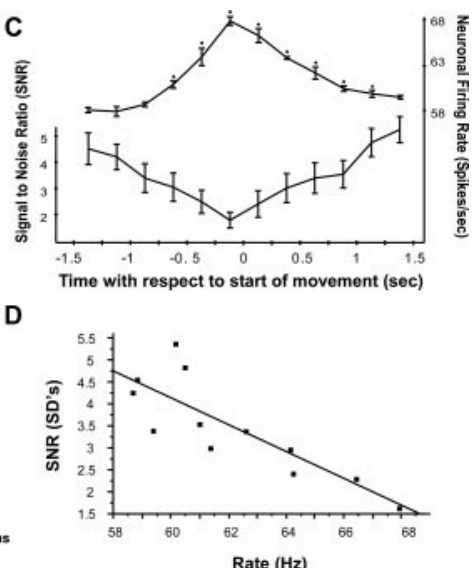

Figure 3. A, Scatter plot of mean power during movement versus at rest. The diagonal line represents the null hypothesis of no change in oscillations during movement. $B$, Mean power of autocorrelograms and cross-correlograms at rest and during movement. Error bars indicate SEM. C, The maximum SNR of the power spectrums and the average neuronal firing rates aligned to the start of movement $(0 \mathrm{msec}) .{ }^{*} p<0.05$, statistically significant changes of neuronal firing rate from baseline (paired $t$ test). $D$, Correlation of firing rates and the power of oscillatory activity.

difficult to evaluate the changes that occur with movement. Ours is one of the few studies to evaluate oscillatory activity by using a structured visual guidance task intraoperatively while recording multiunit activity.

One of the observations of this study is that not all of the patients with significant oscillatory STN activity had resting tremor. This is in contrast to previous work suggesting that oscillatory STN activity is mostly present in patients with moderate resting tremor (Levy et al., 2000). In the study by Levy et al. (2000), six patients had limb tremor during the recording session, two of which had undergone a previous pallidotomy. Furthermore, the patients in that study were at rest and were encouraged to perform mental arithmetic to enhance the tremor. In contrast, none of our subjects had undergone a previous surgery, and our
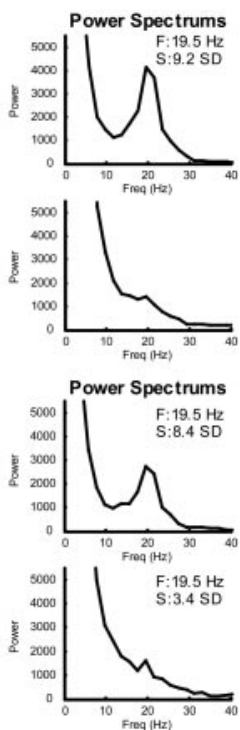

subjects were actively performing the task during recording; hence, there was little or no active tremor. Thus, the differences between the two studies may be attributable to variability between patients, the dynamic nature of tremor, or differences in task conditions.

The relationship between oscillatory activity and the typical 5-7 Hz parkinsonian resting tremor is complex. For example, in primate studies, MPTP-treated macaques exhibit GPi oscillations in the $12-14 \mathrm{~Hz}$ range and have akinesia and rigidity without developing overt tremor (Miller and DeLong, 1987). In contrast, African green monkeys treated with MPTP exhibit both low- and high-frequency oscillations and develop tremor (Bergman et al., 1994; Raz et al., 2000). In previous studies of human subjects, a subset of oscillatory STN cells oscillate at tremor frequency, but the majority of oscillatory cells exhibit high-frequency oscillations $(>10$ $\mathrm{Hz}$ ). In the current study, we focused on neuronal oscillations at a higher frequency $(10-20 \mathrm{~Hz})$, which were not consistently associated with tremor. However, given the limitations of the technique, there may have been lower-frequency oscillations $(<5$ $\mathrm{Hz})$ that were not detected. One recent hypothesis suggests that oscillations in the beta frequency range $(10-30 \mathrm{~Hz})$ interfere with the ability to execute a movement and thus lead to akinesia and bradykinesia, whereas lower-frequency theta band oscillations $(3-10 \mathrm{~Hz})$ are associated more directly with tremor (Dostrovsky and Bergman, 2004). Future studies are needed to analyze further the relationship between low-frequency oscillations and tremor in patients with $\mathrm{PD}$.

The genesis of the oscillatory activity in PD is not completely understood. In normal primates, the motor cortex, premotor cortex, and the striatum exhibit high-frequency oscillations at rest that diminish with movement (Donoghue et al., 1998; Courtemanche et al., 2003). Anatomic studies in primates have demonstrated that the STN receives direct and relatively fast projections from the motor and premotor areas, in addition to the classical projection from the globus pallidus externa (GPe) (Albin et al., 1989; Nambu et al., 1996). Hence, one possibility is that cortical oscillatory activity is transmitted directly to the STN via the projections from the motor and premotor areas and that this activity is suppressed in the normal state but becomes enhanced in the parkinsonian condition. One proposed mechanism is that the higher baseline firing rates observed in the parkinsonian STN are better able to carry this higher-frequency signal (Levy et al., 2002). An alternative possibility is that the oscillations arise from the intrinsic mechanisms in the basal ganglia. Studies in organotypic slices have suggested that the STN and GPe form a feedback system that can engage in synchronized bursting, hence constituting a pacemaker type of activity (Plenz and Kital, 1999). In keeping with this idea, studies of dissociated subthalamic neurons have revealed a resurgent sodium current that can, under the right circumstances, contribute to pacemaker activity (Do and Bean, 2003). This same study demonstrated that the electrical driving of the cells at $75 \mathrm{~Hz}$ led to an inactivation of this resurgent sodium current.

The major findings from the current study suggest that the 
Table 1. Cell distribution and tremor severity by patient

\begin{tabular}{clll}
\hline Patient & Total cells & Oscillatory cells (\% total) & Tremor severity \\
\hline $\begin{array}{llll}\text { Patients with oscillatory STN cells } \\
1\end{array}$ & & \\
2 & 14 & $7(50 \%)$ & Severe \\
3 & 12 & $4(33 \%)$ & Mild \\
4 & 18 & $5(28 \%)$ & None \\
5 & 21 & $1(5 \%)$ & None \\
6 & 24 & $4(17 \%)$ & None \\
7 & 16 & $4(25 \%)$ & Mild \\
Patients without 0 oscillatory STN cells & $1(3 \%)$ & Mild \\
8 & 16 & 0 & \\
9 & 8 & 0 & Moderate \\
10 & 10 & 0 & Mild \\
11 & 16 & 0 & Mild \\
\hline
\end{tabular}

There was no direct relationship noted between the severity of the observed resting tremor and the oscillatory nature of the recordings.

oscillatory activity in the STN of PD patients is dynamic, occurring primarily at rest and dampening with active movements. In agreement with our findings, previous studies relying primarily on recordings from implanted DBS electrodes have demonstrated $15-20 \mathrm{~Hz}$ oscillations in the field potentials, which diminish with movement (Cassidy et al., 2002; Levy et al., 2002). In addition, the current study demonstrates that the decrease in oscillatory activity is tightly correlated with the relative increase in firing rates associated with movement. Furthermore, the increase in firing rate and corresponding decrease in oscillatory activity significantly precede movement initiation.

Together, these lines of evidence suggest the following hypothetical scheme. Movement preparation is associated with increased activity in the premotor and motor areas that is transmitted directly to the STN. This is supported by a study of STN field potentials, which suggested that movement-related potentials can occur well before the actual electromyographic onset of movement (Paradiso et al., 2003). As the firing rates dynamically increase from baseline, the oscillations are progressively dampened, perhaps by inactivation of the resurgent sodium current (Do and Bean, 2003). Ultimately, the oscillations are significantly dampened, and the movement occurs. These observations may represent a natural analog of the effects of high-frequency stimulation. A recent study using MPTP-treated primates demonstrated that high-frequency stimulation of the STN resulted in increased firing rates in its target nucleus, the GPi (Hashimoto et al., 2003). These authors concluded that changes in the firing patterns of neurons in GPe and GPi underlie the beneficial effects of high-frequency stimulation in the STN in PD. Thus, a reasonable hypothesis is that abnormal temporal patterns of activity play a role in the pathogenesis of PD and that disruption of these patterns, whether transiently by cortical activation or chronically by deep brain stimulation, may facilitate movement execution.

\section{References}

Abosch A, Hutchison WD, Saint-Cyr JA, Dostrovsky JO, Lozano AM (2002) Movement-related neurons of the subthalamic nucleus in patients with Parkinson disease. J Neurosurg 97:1167-1172.

Albin RL, Young AB, Penney JB (1989) The functional anatomy of basal ganglia disorders. Trends Neurosci 12:366 -375 .

Alkhani A, Lozano AM (2001) Pallidotomy for parkinson disease: a review of contemporary literature. J Neurosurg 94:43-49.

Bergman H, Wichmann T, Karmon B, DeLong MR (1994) The primate subthalamic nucleus. II. Neuronal activity in the MPTP model of parkinsonism. J Neurophysiol 72:507-520.
Boraud T, Bezard E, Bioulac B, Gross CE (2000) Ratio of inhibited-toactivated pallidal neurons decreases dramatically during passive limb movement in the MPTP-treated monkey. J Neurophysiol 83:1760-1763.

Cassidy M, Mazzone P, Oliviero A, Insola A, Tonali P, Lazzaro VD, Brown P (2002) Movement-related changes in synchronization in the human basal ganglia. Brain 125:1235-1246.

Courtemanche R, Fujii N, Graybiel AM (2003) Synchronous, focally modulated $\beta$-band oscillations characterize local field potential activity in the striatum of awake behaving monkeys. J Neurosci 23:11741-11752.

DBS Study Group (2001) Deep-brain stimulation of the subthalamic nucleus or the pars interna of the globus pallidus in Parkinson's disease. N Engl J Med 345:956-963.

DeLong MR (1990) Primate models of movement disorders of basal ganglia origin. Trends Neurosci 13:281-285.

Do MT, Bean BP (2003) Subthreshold sodium currents and pacemaking of subthalamic neurons: modulation by slow inactivation. Neuron 39:109-120.

Donoghue JP, Sanes JN, Hatsopoulos NG, Gaal G (1998) Neural discharge and local field potential oscillations in primate motor cortex during voluntary movements. J Neurophysiol 79:159-173.

Dostrovsky J, Bergman H (2004) Oscillatory activity in the basal gangliarelationship to normal physiology and pathophysiology. Brain 127:721-722.

Eskandar EN, Assad JA (2002) Distinct nature of directional signals among parietal cortical areas during visual guidance. J Neurophysiol 88:1777-1790.

Eskandar EN, Shinobu LA, Penney JB, Cosgrove GR (2000) Nonmicroelectrode-guided pallidotomy: surgical technique and 2 year results. J Neurosurg 92:375-383.

Glickstein M, Stein J (1991) Paradoxical movement in Parkinson's disease. Trends Neurosci 14:480-482.

Harris KD, Henze DA, Csicsvari J, Hirase H, Buzsaki G (2000) Accuracy of tetrode spike separation as determined by simultaneous intracellular and extracellular measurements. J Neurophysiol 84:401-414.

Hashimoto T, Elder CM, Okun MS, Patrick SK, Vitek J (2003) Stimulation of the subthalamic nucleus changes the firing pattern of pallidal neurons. J Neurosci 23:1916-1923.

Hutchison WD, Allan RJ, Opitz H, Levy R, Dostrovsky JO, Lang AE, Lozano AM (1998) Neurophysiological identification of the subthalamic nucleus in surgery for Parkinson's disease. Ann Neurol 44:622-628.

Lang AE, Lozano AM (1998a) Parkinson's disease. First of two parts. N Engl J Med 339:1044-1053.

Lang AE, Lozano AM (1998b) Parkinson's disease. Second of two parts. N Engl J Med 339:1130-1143.

Lee IH, Assad JA (2003) Putaminal activity for simple reactions or selftimed movements. J Neurophysiol 89:2528 -2537.

Levy R, Hutchison WD, Lozano AM, Dostrovsky JO (2000) High-frequency synchronization of neuronal activity in the subthalamic nucleus of parkinsonian patients with limb tremor. J Neurosci 20:7766-7775.

Levy R, Dostrovsky JO, Lang AE, Sime E, Hutchison WD, Lozano AM (2001) Effects of apomorphine on subthalamic nucleus and globus pallidus internus neurons in patients with Parkinson's disease. J Neurophysiol 86:249-260.

Levy R, Ashby P, Hutchison WD, Lang AE, Lozano AM, Dostrovsky JO (2002) Dependence of subthalamic nucleus oscillations on movement and dopamine in Parkinson's disease. Brain 125:1196-1209.

Miller WC, DeLong MR (1987) Altered tonic activity of neurons in the globus pallidus and subthalamic nucleus in the primate MPTP model of parkinsonism. In: The basal ganglia II (Carpenter MB, Jayraman A, eds), pp 415-427. New York: Plenum.

Nambu A, Takada M, Inase M, Tokuno H (1996) Dual somatotopical representations in the primate subthalamic nucleus: evidence for ordered but reversed body-map transformations from the primary motor cortex and the supplementary motor area. J Neurosci 16:2671-2683.

Paradiso G, Saint-Cyr JA, Lozano AM, Lang AE, Chen R (2003) Involvement of the human subthalamic nucleus in movement preparation. Neurology 61:1538-1545.

Plenz D, Kital ST (1999) A basal ganglia pacemaker formed by the subthalamic nucleus and external globus pallidus. Nature 400:677-682.

Raz A, Vaadia E, Bergman H (2000) Firing patterns and correlations of spontaneous discharge of pallidal neurons in the normal and the tremulous 1-methyl-4-phenyl-1,2,3,6-tetrahydropyridine vervet model of parkinsonism. J Neurosci 20:8559-8571. 